\title{
Molecular investigation of isolates from a multistate polymicrobial outbreak associated with contaminated total parenteral nutrition in Brazil
}

\author{
Marcelo Pillonetto ${ }^{1,2^{*}}$, Lavinia Arend ${ }^{2}$, Suzie M. T. Gomes ${ }^{3}$, Marluce A. A. Oliveira ${ }^{4}$, Loeci N. Timm5 \\ Andreza F. Martins ${ }^{6}$, Afonso L. Barth ${ }^{6}$, Alana Mazzetti ${ }^{1}$, Lena Hersemann ${ }^{7,8}$, Theo H. M. Smits ${ }^{7}$ (D), \\ Marcelo T. Mira ${ }^{1+}$ and Fabio Rezzonico ${ }^{7 \dagger}$
}

\begin{abstract}
Background: Between November 2013 and June 2014, 56 cases of bacteremia (15 deaths) associated with the use of Total Parenteral Nutrition (TPN) and/or calcium gluconate (CG) were reported in four Brazilian states.

Methods: We analyzed 73 bacterial isolates from four states: 45 from blood, 25 from TPN and three from CG, originally identified as Acinetobacter baumannii, Rhizobium radiobacter, Pantoea sp. or Enterobacteriaceae using molecular methods.

Results: The first two bacterial species were confirmed while the third group of species could not be identified using standard identification protocols. These isolates were subsequently identified by Multi-Locus Sequence Analysis as Phytobacter diazotrophicus, a species related to strains from similar outbreaks in the United States in the 1970 's. Within each species, TPN and blood isolates proved to be clonal, whereas the $R$. radiobacter isolates retrieved from CG were found to be unrelated.

Conclusion: This is the first report of a three-species outbreak caused by TPN contaminated with A. baumannii, $R$. radiobacter and $P$. diazotrophicus. The concomitant presence of clonal $A$. baumannii and $P$. diazotrophicus isolates in several TPN and blood samples, as well as the case of one patient, where all three different species were isolated simultaneously, suggest that the outbreak may be ascribed to a discrete contamination of TPN. In addition, this study highlights the clinical relevance of $P$. diazotrophicus, which has been involved in outbreaks in the past, but was often misidentified as $P$. agglomerans.
\end{abstract}

Keywords: Acinetobacter baumannii, Rhizobium radiobacter, Phytobacter diazotrophicus, Pantoea, TPN, Rep-PCR, Bacterial identification

\section{Background}

Total parenteral nutrition (TPN) is an important nutritional supplement for seriously ill patients, especially those incapable of oral or enteral nutrition. TPN are

\footnotetext{
* Correspondence: m.pilonetto@pucpr.br

${ }^{\dagger}$ Marcelo T. Mira and Fabio Rezzonico contributed equally to this work. ${ }^{1}$ Core for Advanced Molecular Investigation, Graduate Program in Health Sciences, School of Medicine, Pontifícia Universidade Católica do Paraná, Curitiba, PR, Brazil

${ }^{2}$ Central Public Health Laboratory - State of Paraná - LACEN/PR, Molecular Bacteriology Division, São José dos Pinhais, PR, Brazil

Full list of author information is available at the end of the article
}

nutritionally rich mixtures and probably the most complex pharmaceutical dosage forms compounded by pharmacists. These infusions, composed by 50 or more constituents, including electrolytes, amino acids, dextrose, lipids and calcium gluconate (CG) [1], are also a favorable growth media for microorganisms [2]. In addition, multiple transfer steps into the same container during preparation increase the risk for microbial contamination [3]. To avoid this, strictly sterile manipulation conditions and total compliance to good manufacturing practices and quality control rules are of paramount importance $[1,4]$.

(c) The Author(s). 2018 Open Access This article is distributed under the terms of the Creative Commons Attribution 4.0 International License (http://creativecommons.org/licenses/by/4.0/), which permits unrestricted use, distribution, and reproduction in any medium, provided you give appropriate credit to the original author(s) and the source, provide a link to the Creative Commons license, and indicate if changes were made. The Creative Commons Public Domain Dedication waiver (http://creativecommons.org/publicdomain/zero/1.0/) applies to the data made available in this article, unless otherwise stated. 
Adoption of these procedures helped TPN-related outbreaks to decline, but adverse events still occur worldwide, usually resulting in severe sepses with high fatality rate, especially in vulnerable individuals [5].

In nine outbreaks reviewed from 1990 to 2006, the mortality following TPN contamination was 48.9\% (19/39) [5]. Incidence of bloodstream infection within patients receiving TPN has been reported between 1.3-39\% [3]. Exposure to TPN is considered an independent risk factor for blood stream infections at neonate intensive care units [6]. The relative risk has been estimated as 4.69 in neonates receiving TPN through central catheter [7].

Several species of microorganisms have been involved in TPN-related outbreaks, including bacteria belonging to the Erwinia herbicola-Enterobacter agglomerans complex (EEC), as well as glucose non-fermenting Gram-negative bacteria, such as Acinetobacter spp. [8-11] and, less frequently, also Rhizobium radiobacter [12].

In November 2013, the municipal autorithies of the City of Curitiba, capital of the state of Paraná, southern Brazil, reported a contamination of TPN bags. The batch was immediately recalled and a retrospective statewide survey was launched, resulting in the identification of 30 cases of TPN-related bacteremia (Additional file 1: Table S1). Case definition was: patient receveing Total Parenteral Nutrition at Hospitals receiving TPN bags from the suspected producers. The finding led the Brazilian Health Surveillance Agency (ANVISA) to trigger a nation-wide epidemiological, sanitary and microbiological investigation, leading to immediate withdraw of the TPN solutions from the suspected manufacturers and closing of the involved TPN pharmaceutical industry for a three-month period. At the same time, 16 additional cases were detected in the southeastern state of Minas Gerais and were linked to a different pharmaceutical industry and one compound pharmacy based on that state, whereby the same precautions were taken by local health autorithies. Additionally, an extensive epidemiological investigation was conducted, tracing the use of the raw material used to manufacture the compound solutions in Paraná and Minas Gerais production plants. The use of the same lot of calcium gluconate in mixtures from both Paraná and Minas Gerais was investigated. The sole calcium gluconate manufacturer industrial plant in Brazil was shut down and sterility tests were launched. In March 2014, one single case of bacteremia was detected in Mogi Mirim, a municipality located southeastern state of São Paulo. From February to June 2014, further nine cases of bacteremia involving use of TPN and/or CG produced in one additional in-house pharmacy (IP) were detected in the southern state of Rio Grande do Sul (Additional file 1: Table S1).

Initial investigation, as performed by manual or automated biochemical methods at the laboratories of the hospitals where the cases were detected, resulted in the identification of mainly three species - "P. agglomerans"/"Pantoea sp.", A. baumannii, and $R$. radiobacter (Additional file 1: Table S2). Alongside, there were sporadic reports of different Enterobacteriaceae species such as Citrobacter amalonaticus, Citrobacter diversus, Kluyvera intermedia, Kluyvera sp. and Enterobacteriaceae spp. Later, in June 2014, $R$. radiobacter was detected in three lots of CG by a private laboratory in Rio Grande do Sul.

Throughout this investigation, a subset of 73 bacterial isolates from blood, TPN and CG were directed to the reference Central Public Health Laboratory of Paraná LACEN for molecular analysis. This subset was composed of all available isolates recovered during the outbreak and some TPN bags that were only partially administered to the patients. Here, we report the results of the microbiological and molecular investigation of this outbreak, with emphasis on the identification of the bacterial species involved and the tracking of the probable sources of contamination.

\section{Methods}

Epidemiological description of the outbreak

Demographic and epidemiological data of the cases were obtained from three different sources: epidemiological reports from municipal and state health autorithies at Paraná, Minas Gerais and Rio Grande do Sul; official reports published by ANVISA; and the Laboratory Information System at LACEN.

\section{Origin of isolates}

Within this study, no clinical samples were handled directly, as we only obtained bacterial isolates from the involved hospitals. All isolates originated from the blood stream of case patients, from TPN bags involved in the treatment of the patients, or from CG vials. These isolates were sent to LACEN in adequate transport containers, together with the microbiological reports produced at the hospitals where the cases were ascertained. From November 2013 through June 2014, a total of 45 isolates from 27 patients were evaluated. Another 25 isolates were recovered directly from TPN (four different manufacturers from four states), while the last three came from three different lots of CG from one single compounding industry (Table 1 and Additional file 1: Table S2).

A microbiological investigation of the pharmaceutical components sterility (such as CG, vitamins, electrolytes, amino acids, and dextrose) used to produce TPN in Paraná was conducted using USP Pharmacopeia Methods (1995). In addition, different equipment parts such as bags, connectors, elastomers and others were also submitted to standard microbiological sterility tests. No microorganisms were recovered from those tests [13]. 
Table 1 Geographic and microbiological data for isolates received during the TPN outbreak in Brazil

\begin{tabular}{|c|c|c|c|}
\hline \multirow[t]{2}{*}{ Source of isolates } & \multicolumn{3}{|l|}{ Bacteria $^{a}$} \\
\hline & EEC (Brenner XII) & R. radiobacter & A. baumannii \\
\hline Blood & 19 (16 pts) & 9 (9 pts) & 17 (11 pts) \\
\hline Total parenteral nutrition & 6 & 14 & 5 \\
\hline Calcium gluconate vials & 0 & 3 & 0 \\
\hline Total & 25 & 26 & 22 \\
\hline \multirow[t]{7}{*}{ Original lab identification } & Pantoea spp. (9) & R. radiobacter (18) & A. baumannii (16) \\
\hline & P. agglomerans (8) & GNFGNB (7) & UNI (6) \\
\hline & C. diversus (4) & UNI (1) & \\
\hline & C. amalonaticus (1) & & \\
\hline & K. intermedia (1) & & \\
\hline & Kluyvera spp. (1) & & \\
\hline & Enterobacteriaceae (1) & & \\
\hline \multicolumn{4}{|l|}{ Geographical origin } \\
\hline \multicolumn{4}{|l|}{ Paraná } \\
\hline Blood & 14 & 5 & 17 \\
\hline TPN & 4 & - & 5 \\
\hline \multicolumn{4}{|l|}{ Minas Gerais } \\
\hline Blood & 5 & - & - \\
\hline TPN & 2 & - & - \\
\hline São Paulo & - & 1 & - \\
\hline Blood & - & - & - \\
\hline TPN & - & - & - \\
\hline \multicolumn{4}{|l|}{ Rio Grande do Sul } \\
\hline Blood & - & 3 & - \\
\hline TPN & - & 14 & - \\
\hline$C G$ & - & 3 & - \\
\hline \multicolumn{4}{|l|}{ Hospitals Involved (cities) } \\
\hline Paraná & $5(3)$ & $4(2)$ & $4(2)$ \\
\hline Minas Gerais & $5(2)$ & - & - \\
\hline São Paulo & - & $1(1)$ & - \\
\hline Rio Grande do Sul & - & $3(1)$ & - \\
\hline
\end{tabular}

${ }^{a}$ Abbreviations: Pts Patients, TPN total parenteral nutrition, CG calcium gluconate, GNFGNB glucose non-fermenting Gram-negative bacteria, UNI unidentified

\section{Microbiological identification}

Strains obtained in this study were routinely grown on MacConkey agar and stored at $-80{ }^{\circ} \mathrm{C}$ as glycerol stocks. Identification of all 73 isolates was performed at LACEN with the Vitek-2 $2^{\circledR}$ platform (Mercy L'Ėtoile, FR) using GN and AST-N 239 cards for species identification and antimicrobial susceptibility testing, respectively. Partial sequences (around $500 \mathrm{bp}$ ) of the $16 \mathrm{~S}$ rRNA gene were obtained using the MicroSEQ $500^{\circ} 16 \mathrm{~S}$ rDNA PCR Kit (Thermo Fisher Scientific - Waltham, MA-USA) and analyzed using Le BIBI https://umr5558-bibiserv.univ-lyon1.fr/lebibi/lebibi.cgi [14] and Sepsi-Test ${ }^{\odot}$ Blast http://www.sepsitest-blast.de/en/ index.html identification tools.

\section{Molecular typing}

Molecular epidemiology analysis was performed using an automated rep-PCR system (Diversilab ${ }^{\oplus}$, BioMerieux) as previously described [15]. Seventeen Enterobacteriaceae (ten from blood and seven from TPN, Fig. 1), eleven Acinetobacter (seven from blood and four from TPN, Fig. 2) and twenty-three Rhizobium (eight from blood, twelve from TPN and three from CG, Fig. 3) 


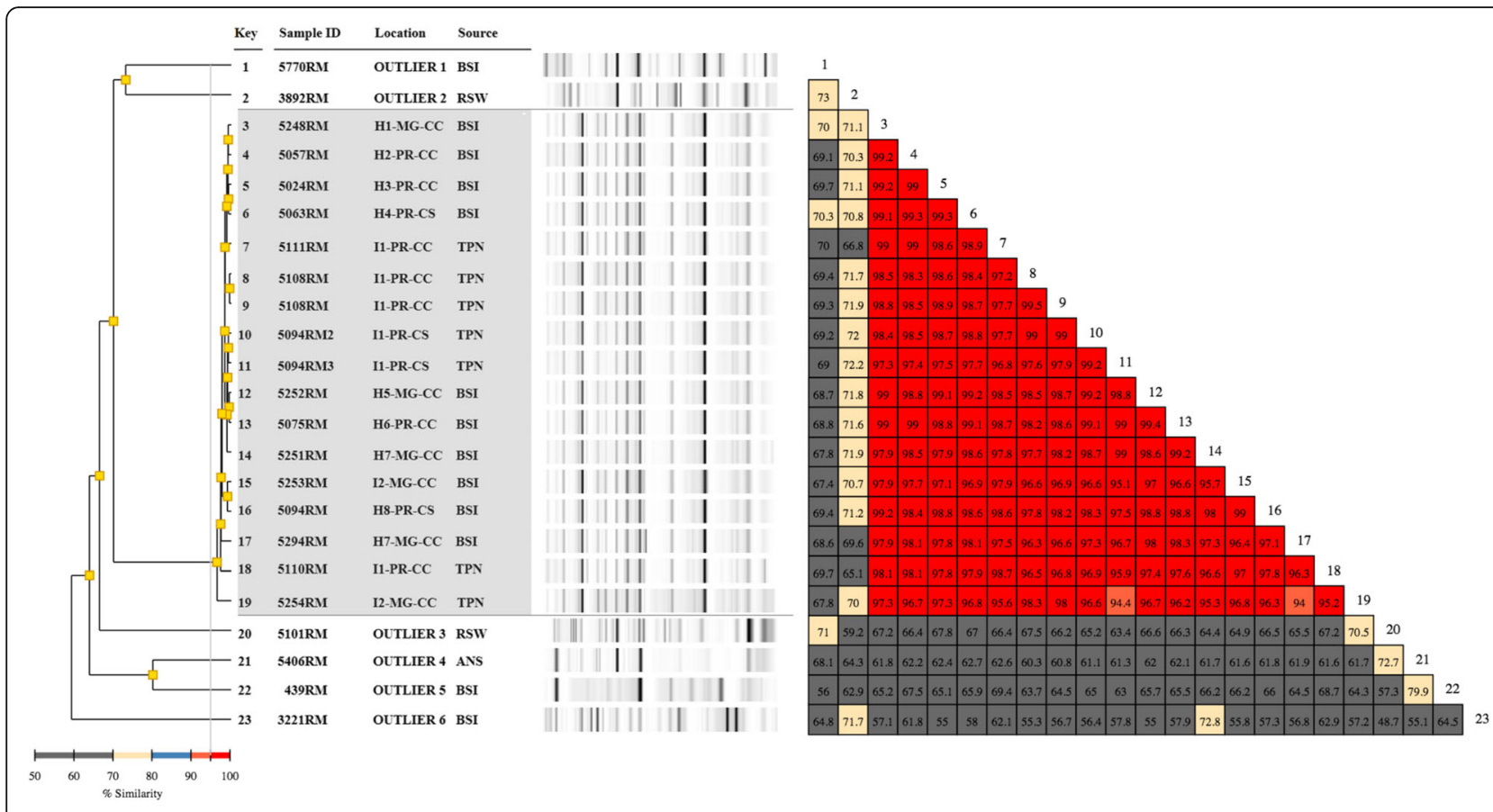

Fig. 1 Rep-PCR based molecular typing and percentage of similarity within Phytobacter diazotrophicus outbreak isolates and outliers. Location: H1 to H7- hospitals numbered sequentially; 11-12 - industries 1 and 2, respectively; MG - Minas Gerais State, PR - Paraná State; CC- capital city; CS - countryside. Source: ANS - ankle secretion; BSI - blood isolates; TPN - total parenteral nutrition; RSW - rectal swab. Shaded area: isolates recovered from the outbreak. The couloured boxes states the percentage of similarity between the two strains. The red boxes indicate higher similarity (above 95\%) between strains meaning isolates are clonal. The orange boxes indicate high similarities (90-95\%) meaning isolates are related -i.e: belong to the same clonal group. Yellow boxes indicate intermediate similarities (70-80\%). The grey boxes indicate similarity is low (50-70\%)

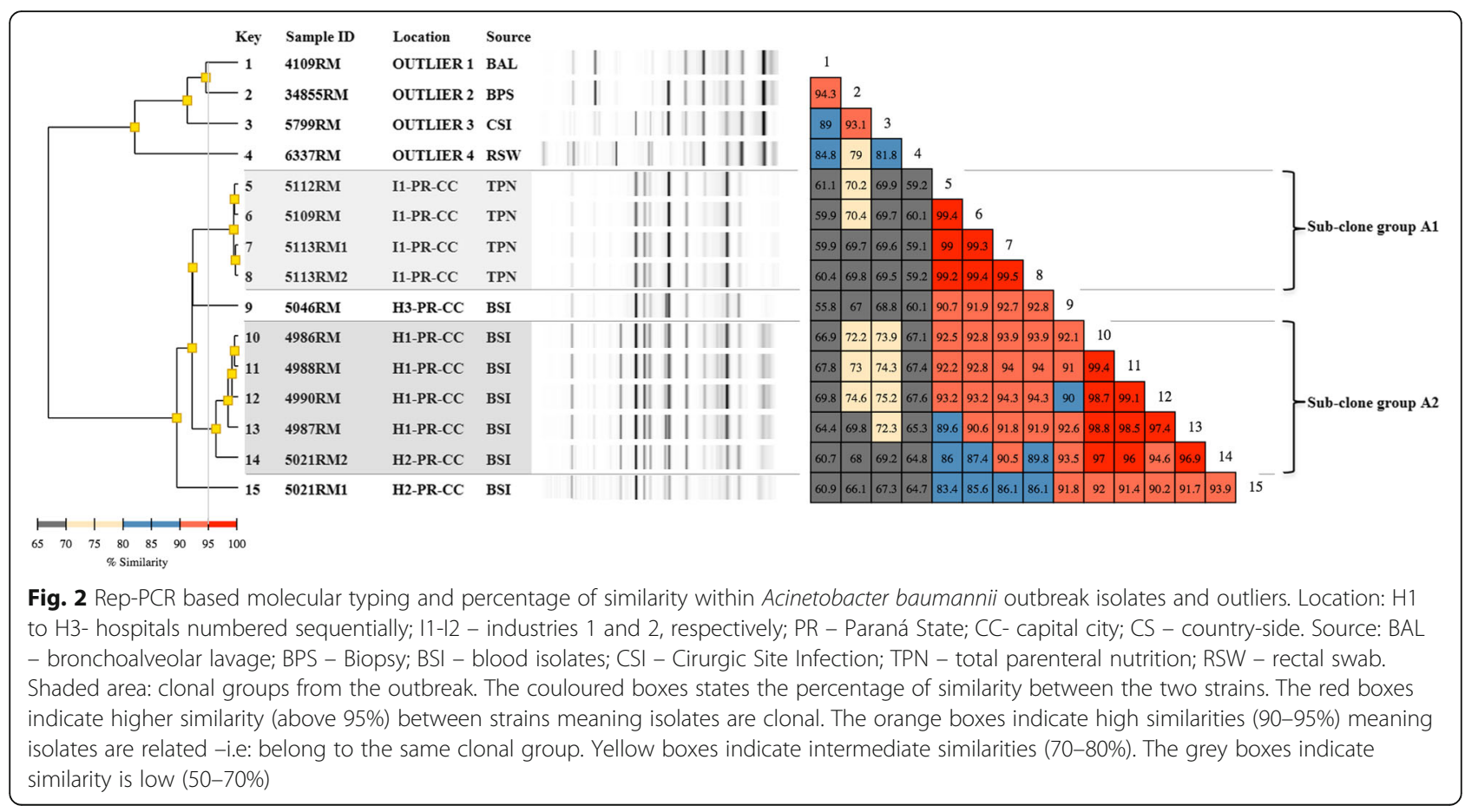




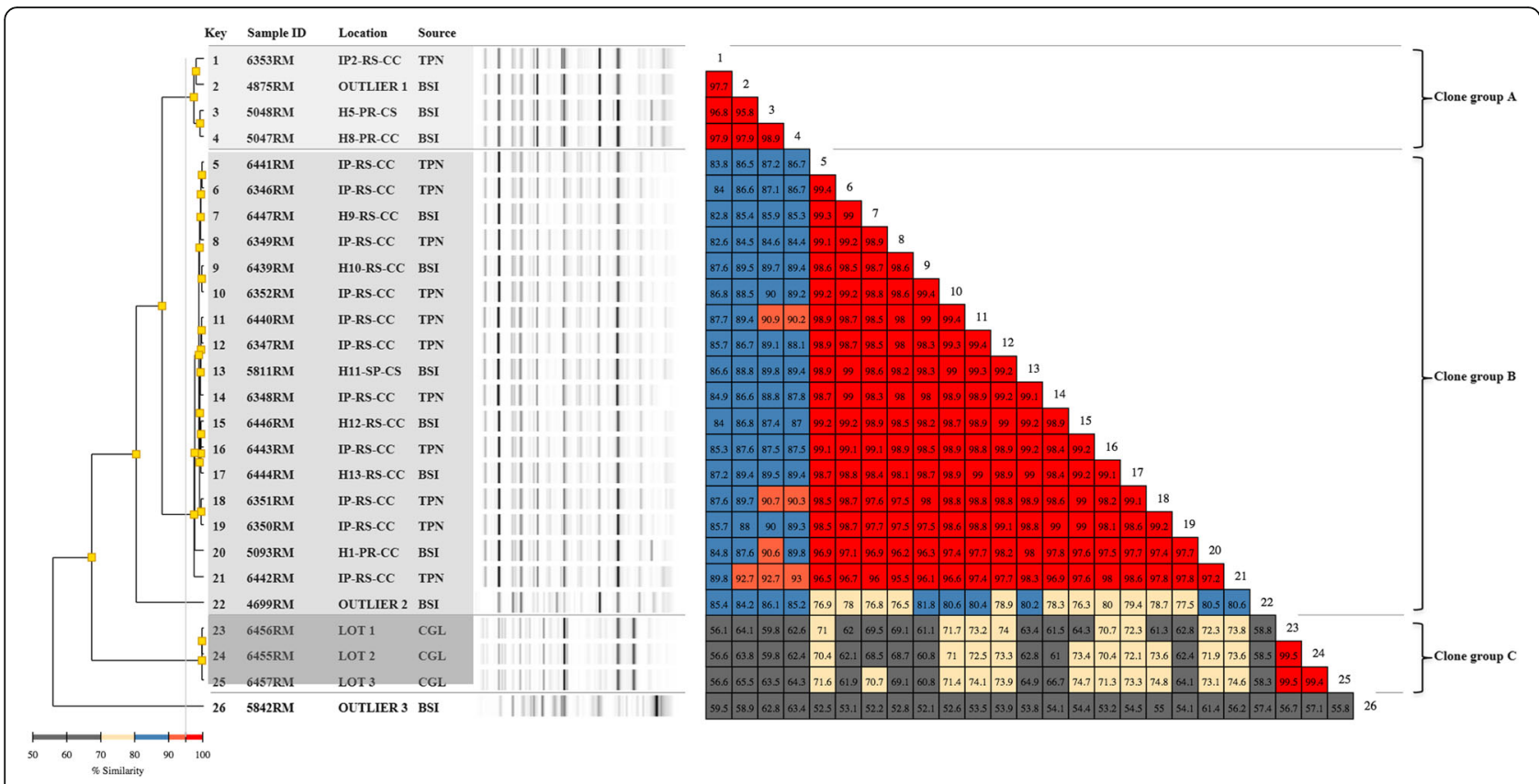

Fig. 3 Rep-PCR based molecular typing and percentage of similarity within Rhizobium radiobacter outbreak isolates and outliers. Location: H1 to H13- hospitals numbered sequentially; IP-In house pharmacy; PR - Paraná State; RS - Rio Grande do Sul State; SP - São Paulo state; CC- capital city; CS - country-side. Source: BSI - blood isolates; CGL - Calcium gluconate vial; TPN - total parenteral nutrition. Shaded area: isolates belonging to the same clone or clonal group. The couloured boxes states the percentage of similarity between the two strains. The red boxes indicate higher similarity (above 95\%) between strains meaning isolates are clonal. The orange boxes indicate high similarities (90-95\%) meaning isolates are related -i.e: belong to the same clonal group. Yellow boxes indicate intermediate similarities (70-80\%). The grey boxes indicate similarity is low $(50-70 \%)$

chosen to represent the widest diversity in isolation source and sample type were submitted to clonal analysis. Isolates sent to LACEN before or after the outbreak period (November 2013-June 2014), but from the same hospitals, if available, were included as outliers for rep-PCR profile comparision, including six "Pantoea spp.", four A. baumannii and three $R$. radiobacter (Figs. 1, 2 and 3).

Rep-PCR results were analyzed using the Pearson correlation statistical method (Diversilab software). Isolates showing a similarity of $90 \%$ or higher were considered as related, whereas if the similarity exceeded 95\%, they were assigned to the same clonal group according to Higgins et al. [16].

\section{Whole-genome sequencing of two Enterobacteriaceae isolates}

A random isolate from TPN (5110RM) [17] and a random isolate from a blood sample (5020RM), both identified by Vitek- $2^{\circ}$ as "Pantoea sp.", were selected for more detailed molecular analysis of all the outbreak isolates primarily assigned to some species within the Enterobacteriaceae. Whole-genome sequencing (WGS) of both isolates was performed on the Illumina MiSeq platform (Illumina Inc., San Diego, USA) and draft genomes were assembled de novo using the SeqMan NGen software included in the DNASTAR Lasergene genomics package version 12 (DNASTAR, Madison, USA) as described elsewhere [17]. Sequences of housekeeping genes atpD, $\operatorname{gyr} B$, infB and $r p o B$ were extracted from WGS data and used to perform MultiLocus Sequence Analysis (MLSA) according to the method described by Brady et al. [18]. A phylogenetic tree was constructed using the concatenated DNA sequences implementing the Neighbor-Joining method in the software MEGA 7 [19]. Average nucleotide identities (ANI) were determined from the subroutine in EDGAR 2.1 [20] after annotation of the genomes in GenDB [21].

\section{Results}

\section{Epidemiological investigation}

We received 45 blood isolates from 15 hospitals of seven different cities in four different states. Additionally, 25 isolates obtained from TPN administered to the patients and three isolates from CG were included in this study (Additional file 1: Table S2). Overall mortality rate was 26.8\% (15/56) (Table 1 and Additional file 1: Table S1). The age distribution of the patients was as follows: 22 were below 1 year of age (medium age: 51.3 days; range: 12 to 240 days), while the remaining were older (range: 4 to 74 years) at the time blood samples were collected. 


\section{Bacterial identification}

Verification of the identity of the 73 isolates at LACEN demonstrated that three different bacterial species were present in the analyzed samples: 22 and 26 isolates were unanimously identified as A. baumannii complex (ABC) and $R$. radiobacter, respectively, both using the Vitek- $2^{\circ}$ platform as well as by $16 \mathrm{~S}$ rRNA gene analyses, thus largely confirming the primary identification performed by the hospital laboratories of origin (Additional file 1: Table S2, accession numbers for archetypal 16S rRNA sequences: A. baumannii isolate 4988RM - MF403059; $R$. radiobacter isolate 5037RM - MF403063). The other 25 isolates could tentatively be assigned to the Enterobacteriaceae family, but were allocated to different species depending on the identification method used (see below).

The additional sterility test of pharmaceutical components in TPN production did not yield any isolates, indicating that no discrete component or equipment hardware was contaminated.

\section{Isolates belonging to the Enterobacteriaceae}

A total of 25 Enterobacteriaceae isolates were referred to LACEN: 19 isolates from blood and six isolates from TPN. The blood isolates originated from 16 different patients, one of which presenting four Enterobacteriaceae isolates obtained on two different days. Fourteen out of the 19 Enterobacteriaceae blood isolates were from five hospitals in three different cities of Paraná; the remaining five isolates were from five hospitals at two different cities of Minas Gerais. Six Enterobacteriaceae isolates were isolated from six different lots of TPN. Four of six Enterobacteriaceae from TPN were from Curitiba (Paraná) and two others from Belo Horizonte (Minas Gerais).

All Enterobacteriaceae isolates were identified at LACEN as "Pantoea sp." using the Vitek-2 platform. On the other hand, partial 16S rRNA gene sequence analysis at the Le BIBI website identified all isolates either as 'Grimontella senegalensis' or as Phytobacter diazotrophicus. Moreover, when the SEPSI-Test Blast website was used to check the identity of partial 16S rRNA genes, all sequences indicated Citrobacter amalonaticus (Additional file 1: Table S2). Manual comparison of the full 16S rRNA gene sequence of isolate 5110RM (a representative isolate from the only clonal group of $P$. diazotrophicus, obtained from TPN) against the $16 \mathrm{~S}$ rRNA gene of the type strain $P$. diazotrophicus DSM $17806^{\mathrm{T}}$ resulted in a high sequence identity $(99,4 \%)$, using BLAST2N (https://blast.ncbi.nlm.nih.gov/Blas t.cgi?PAGE_TYPE=BlastSearch\&BLAST_SPEC=blast2seq\&LINK_LOC=align2seq) [17]. This result was then checked by MLSA analysis using concatenated sequences of housekeeping genes atpD, $\operatorname{gyr} B, \inf B$ and rроB extracted from WGS data of both strains, which confirmed the identification of the isolate as $P$. diazotrophicus [17] (Fig. 4, Additional file 1: Table S3).

Rep-PCR results for the 17 Enterobacteriaceae isolates analyzed showed $95 \%$ or higher similarity among them, thus confirming their clonality (Fig. 1).

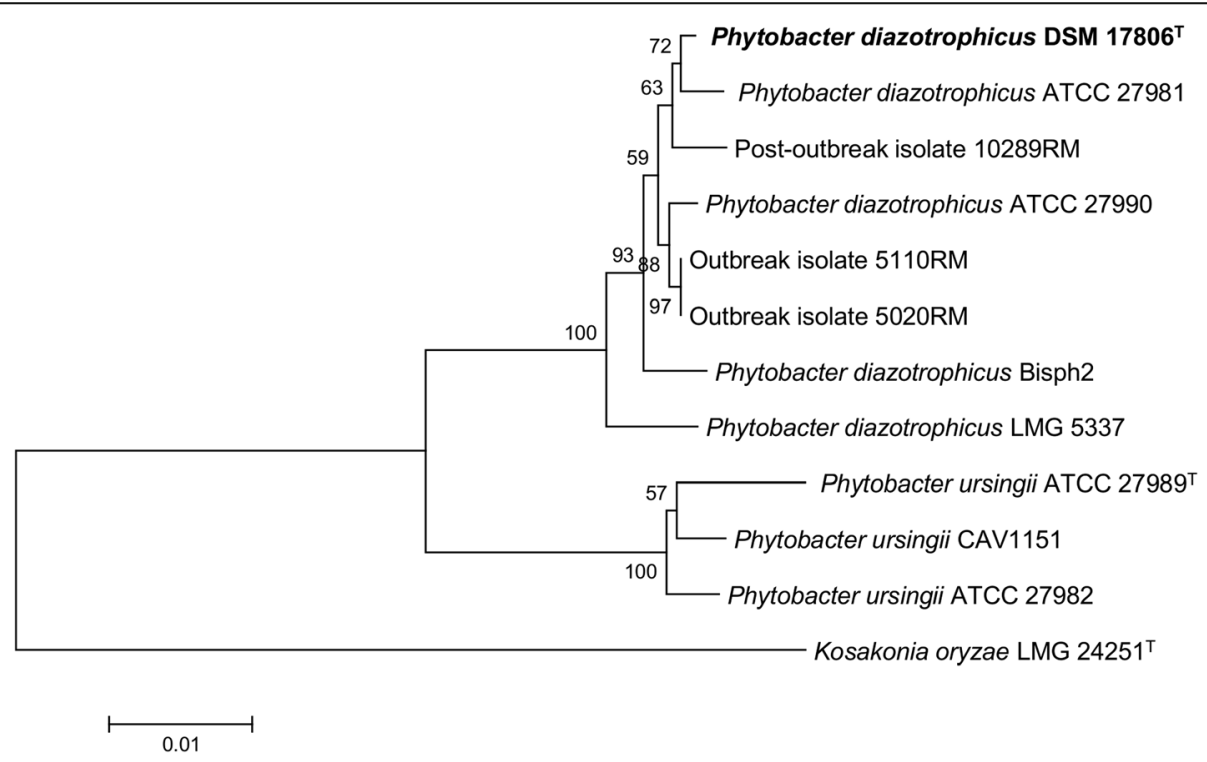

Fig. 4 Position of outbreak isolates 5110RM and 5020RM as well as of post-outbreak isolate 10289RM within P. diazotrophicus as determined by MLSA using concatenated sequences of housekeeping genes $a t p D$, gyrB, infB and rpoB (Brady et al., 2008). The tree was was inferred using the Neighbor-Joining method. Numbers at branching points are bootstrap percentage values (> 50\%) based on 1000 replications. Kosakonia oryzae LMG $24251^{\top}$ as used as outgroup. The scale bar represents $1 \%$ nucleotide substitutions 
Since isolate 5110RM, which was previously assigned to $P$. diazotrophicus [17], is also included in this clone group, we safely can assume that all other Enterobacteriaceae isolates also belong to this species. Sequencing of the genome of a second clinical isolate (5020RM, obtained from blood) allowed the comparison of its MLSA genes. These were identical to those of 5110RM and displayed $99.53 \%$ similarity to type strain $P$. diazotrophicus DSM $17806^{\mathrm{T}}$ [17] (Fig. 4). The genome sequence showed that the two outbreak isolates had average nucleotide identities of 99.99\%, thus further supporting clonality.

Considering the isolates chosen as outliers and as well identified by Vitek-2 as 'Pantoea sp.', two of them (5770RM and 3892RM) showed around 70\% similarity to the clonal outbreak isolates, while the remaining four clustered more distantly (Fig. 1). Ensuing sequence analysis of the MLSA gene set allowed their a more precise assignement of the outliers to a species, whereby only three out of six were effectively found to belong to the genus Pantoea and none was identified as $P$. diazotrophicus (Additional file 1: Table S4).

\section{The A. baumannii isolates}

Twenty-two isolates of A. baumannii complex (ABC) were referred to LACEN, of which 17 originated from ten different patients: eight with single isolates and two with multiple isolates (four and five positive blood cultures, respectively). Five $\mathrm{ABC}$ isolates were originated from five different lots of TPN. All A. baumannii isolates were from Paraná state, and identification was confirmed both by Vitek-2 and partial 16S rRNA gene sequencing (Additional file 1: Table S2).

Four TPN isolates belonged to the same sub-clonal group (A1) with similarity above $99 \%$; one blood isolate (5046RM) showed an average similarity of $92.0 \%$ to sub-clone group A1 (TPN) and 91.5\% to sub-clone group A2 (blood isolates). Within all the latter blood isolates, similarity ranged from 94.6 to $99.4 \%$ based on rep-PCR determinations. Comparison of individual isolates between clone group $\mathrm{A} 1$ and $\mathrm{A} 2$ resulted in a similarity ranging from $86.0 \%$ (5112RM vs. 5021RM2) up to 94.3\% (5113RM vs. 4990RM) (Fig. 2).

While isolates 5113RM1 and 5113RM2 obtained from the same TPN bag proved to be clonal (99.5\% similarity), isolates 5021RM1 and 5021RM2 originating from the same patient failed to reach, albeit by a narrow margin $(93,9 \%)$, the similarity threshold needed to be included within the same clonal group.

\section{The $\mathrm{R}$. radiobacter isolates}

Twenty-six isolates of $R$. radiobacter were referred to LACEN, nine of them originated from blood samples of nine different patients and 14 from different bags of
TPN. No patient presented multiple isolates. Five out of nine isolates from blood samples were from four hospitals at two different cities in Paraná; three isolates were from three hospitals at Porto Alegre (Rio Grande do Sul) and one isolate was from Mogi Mirim (São Paulo State). Additionally, three isolates were obtained from CG vials. All isolates from TPN bags and CG vials were from Porto Alegre. Identification of all $R$. radiobacter isolates was confirmed both by Vitek-2 and partial 16S rRNA gene sequencing (Additional file 1: Table S2). Molecular typing of $R$. radiobacter strains revealed three different groups with internal similarity above $95 \%$ : clonal group A, composed of three outbreak isolates and one outlier; clonal group B with 17 outbreak isolates, and group C, with the three CG isolates. Clonal groups A and B equally contained isolates from blood and TPN and inter-group pairwise comparison of their individual isolates showed a similarity ranging from $82.6 \%$ (6353RM vs. $6349 \mathrm{RM}$ ) to $93.0 \%$ (5047RM vs. $6442 \mathrm{RM}$ ). It is worth to note the different clonality associated to the TPN bags from the two in-house pharmacies (IP) scrutinized, with the sole isolate analyzed from IP2 (6353RM) clustering with clonal group A, whereas all those originating from IP1 could be assigned to clonal group B. Clonal group $\mathrm{C}$, composed solely of CG isolates, was even more distantly related, with a similarity to the other two groups that never exceeded $75 \%$. Two outliers showed up as singlets (Fig. 3).

\section{Connections between the different organisms isolated}

$A$. baumannii and $P$. diazotrophicus were often found in mutual association both in TPN bags as well as in blood samples. Although not all $A$. baumannii isolates were tested by rep-PCR, both clonal sub-groups and one isolate (5021RM1) loosely associated to sub-group A2 could be found among those concomitant with $P$. diazotrophicus. In one case, the two species could be retrieved straight from the TPN bag administered to an infected patient (JVBN), thus reliably confirming the source of infection (Fig. 5). Blood samples from one patient in Paraná (EMP) showed an infection with all three bacteria (A. baumannii, $P$. diazotrophicus and $R$. radiobacter) simultaneously, thus hinting to the possibility of a single tribacterial outbreak. However, in no other sample were $R$. radiobacter isolates found in direct association with A. baumannii or P. diazotrophicus. $R$. radiobacter isolates of the same clonal groups found in patients in Paraná and São Paulo State were retrieved in TPN bags and blood samples from Rio Grande do Sul suggesting a connection between these events, despite the geographical distance.

\section{Discussion}

The molecular investigation of the outbreak led to the identification of isolates belonging to three different 


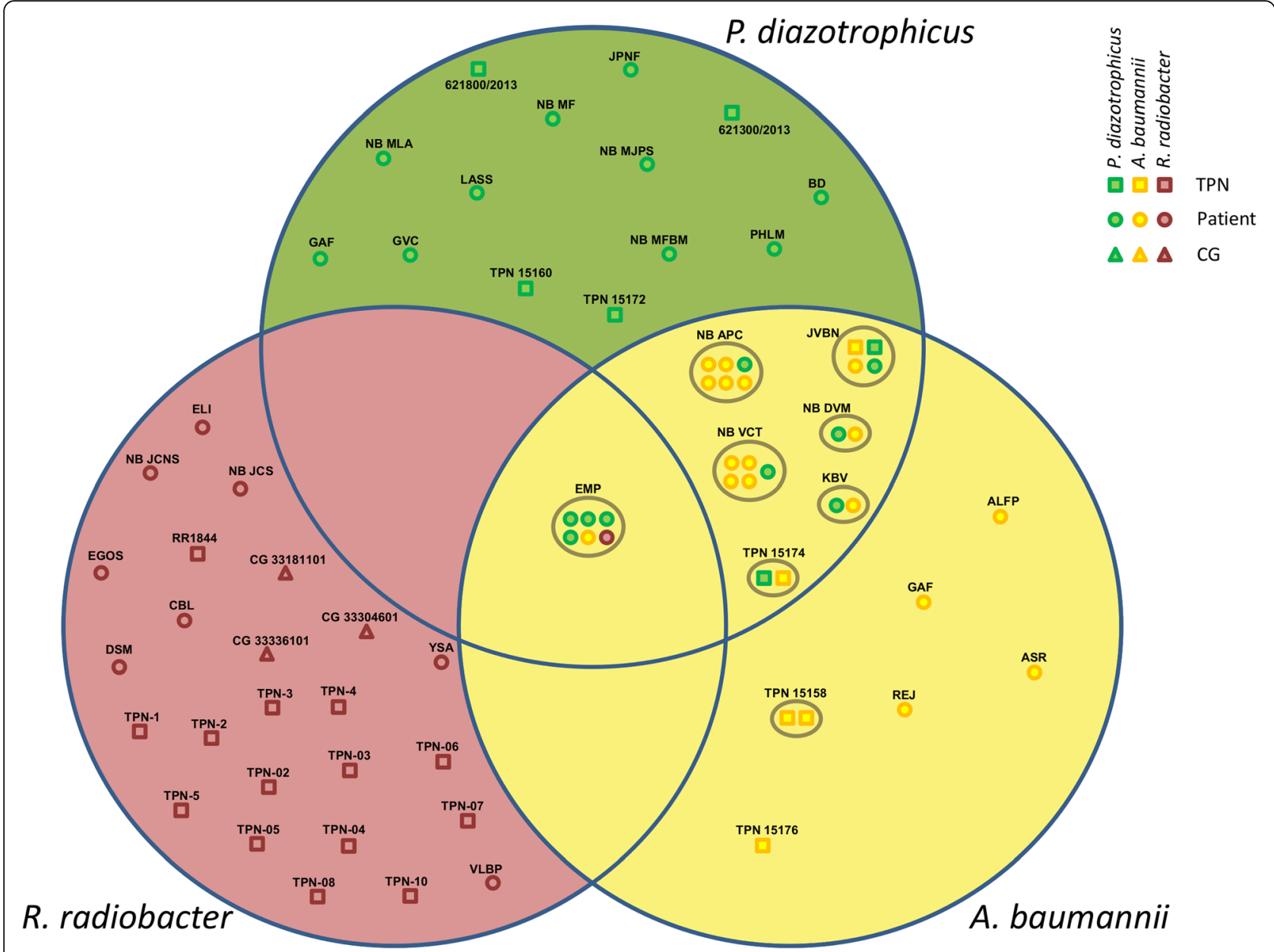

Fig. 5 Venn diagram depicting the affiliation to the three different species involved in the outbreak of all the isolates analyzed at LACEN. Isolates obtained from the same patient, TPN bag or CG vial are regrouped by grey circles. Codes indicate individual patient or sample

bacterial species: $P$. diazotrophicus, A. baumannii and $R$. radiobacter. Molecular typing of the isolates from blood and TPN using rep-PCR revealed clonality for $P$. diazotrophicus and, to lesser extent, for A. baumannii. The fact that both subclonal groups of the latter species were found in association with a clonal infection by $P$. diazotrophicus hints at a single source of contamination for TPN bags (Fig. 5). Furthermore, considering that in $A$. baumannii the similarity between outbreaks isolates rarely fell considerably below $90 \%$ and was dependent on their origin (TPN or blood), the possibility that the observed differences may be due to in-run variations in the rep-PCR [22], rather than to the actual existence of different clones, cannot be discounted (Fig. 2).

On the other hand, three clonal groups could clearly be distinguished within $R$. radiobacter, with calcium gluconate isolates obviously unrelated to those originating from TPN bags and blood. This does not exclude the calcium gluconate as the possible source of contamination, but does not allow drawing any other direct conclusions. One isolate included as outlier in the study, 4875RM, noticeably clustered within clone group A (Fig. 3). Considering that 4875RM was retrieved from a hospital in Paraná in October 2013 (Additional file 1: Table S2), i.e. less than a month before the first confirmed outbreak isolates, it is possible that its existence may be indication of an earlier onset of the outbreak than originally thought.

No definitive source of contamination could be assessed beyond any doubt. The in-depth investigation by the Brazilian Health Surveillance Agency (ANVISA) targeting the various TPN components and their relative lot numbers was not conclusive. Since at least two of the three species are rather uncommon in clinical settings $(R$. radiobacter and $P$. diazotrophicus), it is possible that a concurrent, single-source contamination of TPN mixture may have occurred. This hypothesis is supported by the finding that in at least one patient, all three different species were isolated simultaneously, as well as by blood and/or TPN samples of five patients presenting both $A$. baumannii and $P$. diazotrophicus isolates (Fig. 5). 
R. radiobacter and $P$. diazotrophicus are well known to be plant-associated, water-associated or soil-borne organisms [23, 24]. However, the reported data does not allow a definite conclusion, as calcium gluconate, one of the suspected potential sources investigated, was contaminated by a $R$. radiobacter belonging to a different clone group as the ones found in TPN or isolated from patients (Fig. 3 and Additional file 1: Table S2).

The major problem hindering a definitive identification of the origin of the outbreak is that, probably due to inter-laboratory variations in microbiological sampling procedures, not all species were regularly recovered from all cultured samples, with some laboratories only reporting the prevailing colony type. This could explain why in Paraná all three species could be identified, while in other states only $P$. diazotrophicus or $R$. radiobacter were isolated.

As no single origin could be identified, we cannot completely exclude the possibility that multiple sources were present, indicating a larger systematic problem with TPN. In any case, recurring outbreaks in Brazil and elsewhere in the world indicate that high standards of quality management for TPN solutions are essential. To avoid the recurrence of similar events, these would ideally include more rigid post-production controls for early detection of bacterial contaminations, including the three species identified in this study. Our study also showed that in this case sterility of the different components or equipment hardware used for TPN production was not breached, rather pointing towards the different TPN components as a source of contamination.

The identification issues within the EEC in clinical laboratories were once again exposed by this work. Indeed, at the original hospital laboratories all EEC isolates were incorrectly identified as "Pantoea sp." by Vitek-2 ${ }^{\circledR}$, whereas analysis of partial 16S rRNA gene sequences using routine protocols yielded incoherent results. Only an in-depth molecular investigation implementing MLSA allowed precise identification of the involved Enterobacteriaceae species as $P$. diazotrophicus, an endophytic bacterium originally isolated from wild rice $[17,25]$ that was repeatedly involved in nosocomial outbreaks linked to the use of TPN or injectable solutions in the last five decades [26-34]. This confirms the need to adapt current clinical diagnostics protocol for an improved identification of bacteria belonging to the EEC, which is a recurring issue, especially when biochemical panels or automated systems are employed for species identification $[35,36]$.

\section{Conclusions}

This study highlights the clinical relevance of $P$. diazotrophicus, a species that has been only recently described, but that was frequently misidentified as Pantoea sp. in the past. The complexity of this outbreak investigation, with the concomitant recurrence of three uncommon bacterial species, underpins the importance of standardized protocols for the isolation of all colony types, in order to preserve a maximum of information that is essential to reconstruct the history and origin of an epidemic. Finally, it reinforces the importance of an in-depth molecular characterization before attributing and publishing names of rare and/or atypical species involved in outbreaks in order to avoid misidentifications.

\section{Additional file}

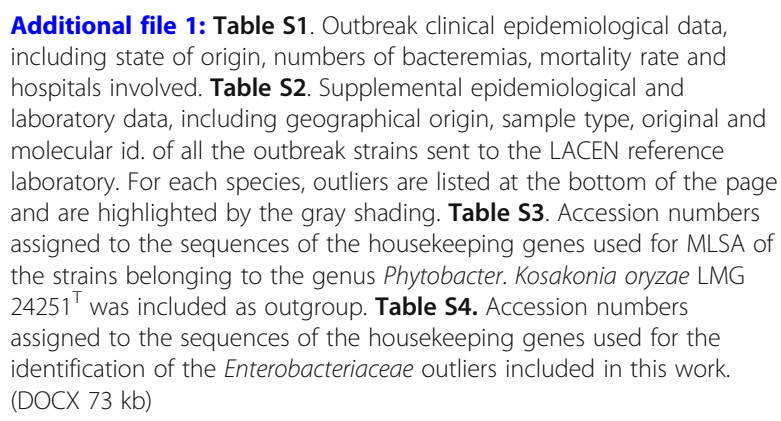

Additional file 1: Table S1. Outbreak clinical epidemiological data, including state of origin, numbers of bacteremias, mortality rate and hospitals involved. Table S2. Supplemental epidemiological and laboratory data, including geographical origin, sample type, original and molecular id. of all the outbreak strains sent to the LACEN reference laboratory. For each species, outliers are listed at the bottom of the page and are highlighted by the gray shading. Table S3. Accession numbers assigned to the sequences of the housekeeping genes used for MLSA of the strains belonging to the genus Phytobacter. Kosakonia oryzae LMG $24251^{\top}$ was included as outgroup. Table S4. Accession numbers assigned to the sequences of the housekeeping genes used for the identification of the Enterobacteriaceae outliers included in this work. (DOCX $73 \mathrm{~kb})$

\section{Abbreviations}

ABC: A. baumannii complex; ANI: Average nucleotide identity; ANVISA: Brazilian Health Surveillance Agency; CG: Calcium gluconate; EEC: Erwinia herbicola-Enterobacter agglomerans complex; IP: In-house pharmacy; LACEN: Central Public Health Laboratory of Paraná; MLSA: MultiLocus Sequence Analysis; TPN: Total parenteral nutrition; WGS: Whole-genome sequencing

\section{Acknowledgements}

We are thankful to Helena R. S. D'Espindula and Monica Dalmann at PUCPR and Karin O. Kalluf at LACEN for technical assistance.

\section{Funding}

This study was supported by the Central Public Health Laboratory of Paraná (LACEN/PR), the Core for Advanced Molecular Investigation at PUCPR, the FLASH Research Programme Project SAMBA funded by the Swiss State Secretariat for Education, Research and Innovation (SERI), and the Life Science and Facility Management Department at Zurich University for Applies Sciences (ZHAW). None of the funding agencies were involved in design of the study, in the collection, analysis and interpretation of data, nor in the writing of the manuscript.

\section{Availability of data and materials}

GenBank accession numbers of MLSA data determined in this study are included in this published article in Additional File 1: Table S3 and Table S4. The genome sequences analyzed during the current study are available from the corresponding author on reasonable request.

\section{Authors' contributions}

MP, THMS, MTM and FR designed the study. MP, LA, MAAO, LNT, AFM, ALB and AM performed bacterial isolation and phenotypic identification experiments. MP, LA, AFM, ALB, LH, THMS and FR performed molecular and genomic experiments. MAAO, LNT and SMTG provided epidemiological data. MP, THMS and FR analyzed data. MP, THMS, MTM and FR wrote the manuscript. All authors read and approved the final version of the manuscript. 


\section{Ethics approval and consent to participate}

As in this study, we only handled bacterial isolates obtained from the involved hospitals, an ethical approval for work with clinical samples was not required.

\section{Consent for publication}

Not applicable.

\section{Competing interests}

The authors declare that they have no competing interests.

\section{Publisher's Note}

Springer Nature remains neutral with regard to jurisdictional claims in published maps and institutional affiliations.

\section{Author details}

${ }^{1}$ Core for Advanced Molecular Investigation, Graduate Program in Health Sciences, School of Medicine, Pontifícia Universidade Católica do Paraná, Curitiba, PR, Brazil. ${ }^{2}$ Central Public Health Laboratory - State of Paraná LACEN/PR, Molecular Bacteriology Division, São José dos Pinhais, PR, Brazil.

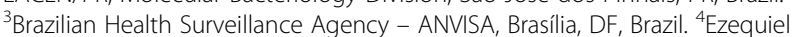
Dias Foundation, Central Public Health Laboratory - State of Minas Gerais, Bacteriology Division, Belo Horizonte, MG, Brazil. ${ }^{5}$ Central Public Health Laboratory - State of Rio Grande do Sul, Bacteriology Division, Porto Alegre, RS, Brazil. ' ${ }^{6}$ Research Laboratory on Bacterial Resistance (LABRESIS), Federal University of Rio Grande do Sul, Hospital de Clínicas, Porto Alegre, RS, Brazil. ${ }^{7}$ Environmental Genomics and Systems Biology Research Group, Institute of Natural Resource Sciences (IUNR), Zurich University of Applied Sciences ZHAW, Wädenswil, Switzerland. ${ }^{8}$ Present Address: Scientific Computing Facility, Max Planck Institute of Molecular Cell Biology and Genetics, Pfotenhauerstrasse 108, 01307 Dresden, Germany.

Received: 30 November 2017 Accepted: 31 July 2018

Published online: 13 August 2018

\section{References}

1. Driscoll D. Compounding TPN admixtures: then and now. J Parenter Enter Nutr. 2003;27:433-8.

2. Kuwahara T, Kaneda S, Shimono K, Inoue Y. Growth of microorganisms in total parenteral nutrition solutions without lipid. Int J Med Sci. 2010;7:43-7.

3. Turpin RS, Canada T, Rosenthal V, Nitzki-George D, Liu FX, Mercaldi CJ, et al. Bloodstream infections associated with parenteral nutrition preparation methods in the United States: a retrospective, large database analysis. J Parenter Enter Nutr. 2012;36:169-76.

4. Gupta N, Hocevar SN, Moulton-Meissner HA, Stevens KM, Mclntyre MG, Jensen B, et al. Outbreak of Serratia marcescens bloodstream infections in patients receiving parenteral nutrition prepared by a compounding pharmacy. Clin Infect Dis. 2014:59:1-8.

5. Vonberg RP, Gastmeier P. Hospital-acquired infections related to contaminated substances. J Hosp Infect. 2007:65:15-23.

6. Távora ACVCF, Castro AB, Militão MAM, Girão JE, de CB RK, LGF T. Risk factors for nosocomial infection in a Brazilian neonatal intensive care unit. Braz J Infect Dis. 2008;12:75-9.

7. Perlman SE, Saiman L, Larson EL. Risk factors for late-onset health careassociated bloodstream infections in patients in neonatal intensive care units. Am J Infect Control. 2007;35:177-82.

8. von Dolinger de Brito D, Oliveira EJ, Abdallah VOS, da Costa Darini AL, Filho PPG. An outbreak of Acinetobacter baumannii septicemia in a neonatal intensive care unit of a university hospital in Brazil. Braz J Infect Dis. 2005;9:301-9.

9. Kilic A, Li H, Mellmann A, Basustaoglu AC, Kul M, Senses Z, et al. Acinetobacter septicus sp. nov. association with a nosocomial outbreak of bacteremia in a neonatal intensive care unit. J Clin Microbiol. 2008;46:902-8.

10. McGrath EJ, Chopra T, Abdel-Haq N, Preney K, Koo W, Asmar Bl, et al. An outbreak of carbapenem-resistant Acinetobacter baumannii infection in a neonatal intensive care unit: investigation and control. Infect Control Hosp Epidemiol. 2011:32:34-41.

11. Ng PC, Herrington RA, Beane CA, Ghoneim ATM, Dear PRF. An outbreak of acinetobacter septicaemia in a neonatal intensive care unit. J Hosp Infect. 1989;14:363-8.
12. Zhao VM, Griffith DP, Blumberg HM, Dave NJ, Battey CH, McNally TA, et al. Characterization of post-hospital infections in adults requiring home parenteral nutrition. Nutrition. 2013;29:52-9.

13. Pharmacopeia, United States. US Pharmacopeial convention. Rockville: X. X. I. I; 1995.

14. Devulder G, Perrière G, Baty F, Flandrois JP. BIBI, a bioinformatics bacterial identification tool. J Clin Microbiol. 2003:41:1785-7.

15. Cieslinski JM, Arend L, Tuon FF, Silva EP, Ekermann RGS, Dalla-Costa LM, et al. Molecular epidemiology characterization of OXA-23 carbapenemaseproducing Acinetobacter baumannii isolated from 8 Brazilian hospitals using repetitive sequence-based PCR. Diagn Microbiol Infect Dis. 2013;77:337-40.

16. Higgins PG, Janssen K, Fresen MM, Wisplinghoff H, Seifert H. Molecular epidemiology of Acinetobacter baumannii bloodstream isolates obtained in the United States from 1995 to 2004 using rep-PCR and multilocus sequence typing. J Clin Microbiol. 2012;50:3493-500.

17. Pillonetto M, Arend L, Faoro H, D'Espindula HRS, Jochen B, Smits THM, et al. Emended description of the genus Phytobacter, its type species Phytobacter diazotrophicus (Zhang 2008) and description of Phytobacter ursingii sp. nov. Int J Syst Evol Microbiol. 2018;68:176-84.

18. Brady C, Cleenwerck I, Venter S, Vancanneyt M, Swings J, Coutinho T. Phylogeny and identification of Pantoea species associated with plants, humans and the natural environment based on multilocus sequence analysis (MLSA). Syst Appl Microbiol. 2008;31:447-60.

19. Kumar S, Stecher G, Tamura K. MEGA7: molecular evolutionary genetics analysis version 7.0 for bigger datasets brief communication. Mol Biol Evol. 2016;33(7):1870-4. https://doi.org/10.1093/molbev/msw054. Epub 2016 Mar 22.

20. Blom J, Kreis J, Spänig S, Juhre T, Bertelli C, Ernst C, et al. EDGAR 2.0 : an enhanced software platform for comparative gene content analyses. Nucleic Acids Res. 2016;44(W1):W22-8. https://doi.org/10.1093/nar/gkw255. Epub 2016 Apr 20.

21. Meyer F, Goesmann A, McHardy AC, Bartels D, Bekel T, Clausen J, et al. GenDB-an open source genome annotation system for prokaryote genomes. Nucleic Acids Res. 2003;31:2187-95.

22. Healy M, Huong J, Bittner T, Lising M, Frye S, Raza S, et al. Microbial DNA typing by automated repetitive-sequence-based PCR. J Clin Microbiol. 2005; 43:199-207.

23. Brenner DJ, Fanning GR, Leete Knutson JK, Steigerwalt AG, Krichevsky MI. Attempts to classify Herbicola group-Enterobacter agglomerans strains by deoxyribonucleic acid hybridization and phenotypic tests. Int J Syst Bacteriol. 1984;34:45-55.

24. Freney J, Gruer LD, Bornstein N, Kiredjian M, Guilvout I, Letouzey MN, et al. Septicemia caused by Agrobacterium sp. J Clin Microbiol. 1985;22:683-5.

25. Zhang GX, Peng GX, Wang ET, Yan H, Yuan QH, Zhang W, et al. Diverse endophytic nitrogen-fixing bacteria isolated from wild rice Oryza rufipogon and description of Phytobacter diazotrophicus gen. nov. sp. nov. Arch Microbiol. 2008;189:431-9.

26. Maki DG, Martin WT. Nationwide epidemic of septicemia caused by contaminated infusion products. IV. Growth of microbial pathogens in fluids for intravenous infusion. J Infect Dis. 1975;131:267-72.

27. Maki DG, Rhame FS, Mackel DC, Bennett JV. Nationwide epidemic of septicemia caused by contaminated intravenous products. Am J Med. 1976; 60:471-85.

28. Bergman KA, Arends JP, Schölvinck EH. Pantoea agglomerans septicemia in three newborn infants. Pediatr Infect Dis J. 2007;26:453-4.

29. Bicudo EL, Macedo VO, Carrara MA, Castro FFS, Rage RI. Nosocomial outbreak of Pantoea agglomerans in a pediatric urgent care center. Braz J Infect Dis. 2007;11:281-4.

30. Abdullah A, Sabbir Alam SM, Sultana M, Hossain MA. BioCluster: tool for identification and clustering of Enterobacteriaceae based on biochemica data. Genomics Proteomics Bioinformatics. 2015;13:192-9.

31. Cheng A, Liu CY, Tsai HY, Hsu MS, Yang CJ, Huang YT, et al. Bacteremia caused by Pantoea agglomerans at a medical center in Taiwan, 2000-2010. J Microbiol Immunol Infect. 2013;46:187-94.

32. Boszczowski Í, Nóbrega de Almeida Júnior J, Peixoto de Miranda É, Pinheiro Freire M, Guimarães T, Chaves CE, et al. Nosocomial outbreak of Pantoea agglomerans bacteraemia associated with contaminated anticoagulant citrate dextrose solution: new name, old bug? J Hosp Infect. 2012:80, 255-258.

33. Garrett DO, McDonald LC, Wanderley A, Wanderley C, Miller P, Carr J, et al. An outbreak of neonatal deaths in Brazil associated with contaminated intravenous fluids. J Infect Dis. 2002;186:81-6. 
34. Campos LC, Lobianco LF, Seki LM, Santos RMR, Asensi MD. Outbreak of Enterobacter hormaechei septicaemia in newborns caused by contaminated parenteral nutrition in Brazil. J Hosp Infect. 2007;66:95-7.

35. Rezzonico F, Smits THM, Duffy B. Misidentification slanders Pantoea agglomerans as a serial killer. J Hosp Infect. 2012;81:137-9.

36. Rezzonico F, Stockwell VO, Tonolla M, Duffy B, Smits THM. Pantoea clinical isolates cannot be accurately assigned to species based on metabolic profiling. Transpl Infect Dis. 2012;14:220-1.

Ready to submit your research? Choose BMC and benefit from:

- fast, convenient online submission

- thorough peer review by experienced researchers in your field

- rapid publication on acceptance

- support for research data, including large and complex data types

- gold Open Access which fosters wider collaboration and increased citations

- maximum visibility for your research: over $100 \mathrm{M}$ website views per year

At BMC, research is always in progress.

Learn more biomedcentral.com/submissions 